\title{
Yếu tố ảnh hưởng đến ý định mua thực phẩm hữu cơ của người tiêu dùng tại Thành phố Hồ Chí Minh
}

\section{Factors affecting intention to purchase organic food among customers in Ho Chi Minh City}

\author{
Nguyễn Thảo Nguyên ${ }^{1 *}$, Lê Thị Trang ${ }^{1}$ \\ ${ }^{1}$ Trường Đại học Kinh tế Thành phố Hồ Chí Minh, Việt Nam \\ *Tác giả liên hệ, Email: nguyennt@ueh.edu.vn
}

\section{THÔNG TIN}

DOI: $10.46223 /$ HCMCOUJS. econ.vi.16.1.1387.2021

Ngày nhận: 13/05/2020

Ngày nhận lại: 07/08/2020

Duyệt đăng: 18/08/2020

Tù khóa:

thực phẩm hữu cơ, ý thức sức khỏe, an toàn thực phẩm, chất lượng
Keywords:

organic food, health consciousness, food safety, quality

\section{TÓM TÁ́T}

Ngày nay, khái niệm thực phẩm hữu cơ đã không còn xa lạ đối với người tiêu dùng. Mọi người bắt đầu nhận thức được tầm quan trọng của việc tiêu thụ thực phẩm tự nhiên, sạch và tốt cho sức khỏe là rất quan trọng trong cuộc sống hàng ngày. Nghiên cứu nhằm xác định các yếu tố như ý thức về an toàn thực phẩm, sức khỏe, chất lượng, môi trường và giá cả và tác động của nó đối với ý định mua hàng của người tiêu dùng tại Thành phố Hồ Chí Minh. Những phát hiện của nghiên cứu chỉ ra rằng trong số năm yếu tố trên, ý thức về an toàn thực phẩm và sức khỏe ảnh hưởng mạnh đến ý định mua hàng của khách hàng. Các yếu tố còn lại cũng được chứng minh ảnh hưởng ít đến ý định mua hàng của khách hàng. Vì ý định mua là một chỉ số quan trọng về tiêu thụ thực phẩm hữu cơ trong tương lai, các nhà hoạch định chính sách và kinh doanh có thể dựa vào những kết quả này khi cố gắng thúc đẩy thị trường tiêu thụ thực phẩm hữu cơ ở Thành phố Hồ Chí Minh.

\section{ABSTRACT}

Nowadays, the concept of organic food is no stranger to consumers. People are beginning to be aware of the importance of consuming natural, clean and healthy food that is so important in everyday life. The research aims to identify factors such as awareness of food safety, health consciousness, quality, environment and price and its impact on the customer purchase intention in Ho Chi Minh City. The findings of the study indicated that out of the five factors mentioned above, food safety and health consciousness influenced the customer purchase intention. The remaining factors have also been shown to have little effect on customers' buying intentions. As intention to purchase is an important indicator of future organic food consumption, policymakers and marketers may draw on these results when attempting to promote the organic food consumption market in Ho Chi Minh City. 


\section{Giới thiệu}

Thực phẩm hữu cơ được định nghĩa là thực phẩm được sản xuất mà không có thuốc diệt cỏ, thuốc trừ sâu, kháng sinh, phân bón vô cơ và hormone tăng trưởng theo Honkanen (Honkanen, Verplanken, \& Olsen, 2006). Các nguồn tài liệu khác nhau đưa các định nghĩa khác nhau về thực phẩm hữu cơ, nhưng gần như tất cả các định nghĩa đều dựa trên các thuộc tính như an toàn, dinh dưỡng, tính chất quan trọng, và tự nhiên (Kahl et al., 2012). Thực phẩm hữu cơ đang trở nên phổ biến ở các nước phát triển và đang phát triển. Người tiêu dùng đã quan tâm nhiều đến thực phẩm hữu cơ vì chúng được sản xuất mà không sử dụng thuốc trừ sâu, các nguyên vật liệu liên quan đến nông nghiệp khác có hại cho sức khỏe con người. Các vụ bê bối về thực phẩm cũng được các phương tiện truyền thông quan tâm và cảnh báo đã ảnh hưởng không nhỏ đến lựa chọn của người tiêu dùng. Năm 2019, toàn quốc ghi nhận 76 vụ ngộ độc thực phẩm làm gần 2.000 người mắc, 1.918 người đi viện và 8 trường hợp tử vong. So với năm 2018 , số vụ giảm 32 vụ (29,6\%), số mắc giảm 1.478 người (42,6\%), số đi viện giảm 1.135 người $(37,2 \%)$, số tử vong giảm 9 người $(52,9 \%)$. Do đó, nhu cầu về thực phẩm hữu cơ tăng cao thúc đẩy nhà sản xuất chuyển từ thực phẩm thông thường sang thực phẩm hữu cơ. Nghiên cứu này nhằm mục đích điều tra các yếu tố mô tả ý định của người tiêu dùng khi mua thực phẩm hữu cơ. Các yếu tố, chẳng hạn như ý thức về sức khỏe, an toàn thực phẩm hay ý thức về môi trường... đã được chọn để nghiên cứu.

Người tiêu dùng tiếp nhận thông tin từ các phương tiện truyền thông chính thống và cả không chính thống, đó là một loại thông tin không cân xứng về thực phẩm hữu cơ và an toàn thực phẩm. Mặc dù thông tin được cung cấp góp phần tăng cường ý định mua hàng của người tiêu dùng, người tiêu dùng vẫn khó có thể phân biệt các thuộc tính của thực phẩm hữu cơ với thực phẩm thông thường. Tình trạng này có thể ảnh hưởng tiêu cực đến ý định mua hàng của người tiêu dùng và nhu cầu thực phẩm hữu cơ trên thị trường. Không hiểu được các yếu tố thúc đẩy ý định mua thực phẩm hữu cơ, những nỗ lực tiếp cận thị trường thực phẩm hữu cơ là chưa đầy đủ. Việc xác định các yếu tố ảnh hưởng đến ý định của người tiêu dùng mua thực phẩm hữu cơ và kết quả nghiên cứu này sẽ rất hữu ích cho các bên liên quan. Những phát hiện này sẽ cung cấp bằng chứng về động cơ của người tiêu dùng để mua thực phẩm hữu cơ bên cạnh các bằng chứng hiện tại. Hơn nữa, các yếu tố này sẽ có lợi cho các bên liên quan để thiết lập các chiến lược thị trường phù hợp để phát triển nhu cầu dài hạn cho các sản phẩm thực phẩm này. Nghiên cứu đã sử dụng Thành phố Hồ Chí Minh làm mẫu vì thành phố này là 1 trong 2 thị trường tiêu thụ thực phẩm hữu cơ lớn của ngành công nghiệp thực phẩm Việt Nam, nó cho thấy những thay đổi tích cực đối với tiêu dùng sản phẩm thực phẩm xanh khi có sự gia tăng mối quan tâm của người tiêu dùng để có được một sản phẩm thực phẩm lành mạnh và thân thiện với môi trường. Điều này cho thấy có một nhu cầu cấp thiết để xác định các yếu tố quan trọng ảnh hưởng đến ý định mua sản phẩm thực phẩm hữu cơ, đặc biệt là đối với người tiêu dùng trẻ tuổi (Hassan, Yee, \& Ray, 2015). Do đó, mục tiêu của nghiên cứu này là xác định các yếu tố ảnh hưởng đến ý định mua hàng của người tiêu dùng đối với sản phẩm thực phẩm hữu cơ.

\section{Cơ sở lý thuyết}

Tổng hợp các nghiên cứu trước chỉ ra rằng có 5 yếu tố: an toàn thực phẩm, ý thức về sức khỏe, ý thức môi trường, chất lượng và giá cả tác động đến ý định mua thực phẩm hữu cơ.

\section{An toàn thực phẩm}

An toàn thực phẩm liên quan đến việc xử lý an toàn thực phẩm từ khi được trồng/nuôi, đóng gói, phân phối và chuẩn bị để ngăn ngừa các bệnh do thực phẩm gây ra (Cerjak, Mesić, Kopić, Kovačić, \& Markovina, 2010). An toàn thực phẩm là trách nhiệm của những người xử lý 
và chuẩn bị thực phẩm thương mại để giao cho người tiêu dùng và người tiêu dùng chuẩn bị và ăn thực phẩm trong nhà của họ. Một số nghiên cứu cũng đã phân tích nhu cầu của người tiêu dùng đối với các chương trình ghi nhãn thịt đảm bảo chất lượng và an toàn (Bernués, Olaizola, \& Corcoran, 2003); (Yeung \& Yee, 2003). An toàn thực phẩm và cách nó ảnh hưởng đến sức khỏe đã trở thành mối quan tâm ngày càng tăng ở hầu hết các nơi trên thế giới (Scarpa \& Thiene, 2011). Một cuộc khảo sát quốc tế cho thấy phần lớn người dân ở 19 trên 35 quốc gia cảm thấy rằng thực phẩm của họ kém an toàn hơn so với 10 năm trước (Reid, 2000). Ngày nay, người tiêu dùng đòi hỏi thông tin đáng tin cậy hơn về thực phẩm họ mua, đặc biệt là về tính hợp lệ của các loại thịt đảm bảo an toàn thực phẩm (Verbeke \& Viaene, 1999). Tóm lại, an toàn thực phẩm có khả năng củng cố ý định mua hàng của người tiêu dùng đối với thực phẩm hữu cơ.

$H_{1}$ : An toàn thực phẩm càng được đánh giá cao sẽ thúc đẩy ý định mua thực phẩm hũu cơ của người tiêu dùng

\section{Ý thức về sức khỏe}

Người tiêu dùng có ý thức về sức khỏe cao đang có xu hướng tìm kiếm và tham gia vào các hoạt động, lối sống lành mạnh. Hơn nữa, theo (Ahmad, Omar, \& Rose, 2015), người tiêu dùng có ý thức về sức khỏe mua sản phẩm xanh vì nó sẽ mang lại tác động không chỉ cho sức khỏe của chính họ mà còn cho môi trường. Điều này cũng có thể áp dụng cho mô hình tiêu thụ thực phẩm hữu cơ, nơi người tiêu dùng rất đặc biệt và nhận thức về an toàn thực phẩm vì họ cần đảm bảo thực phẩm họ ăn không gây hại cho sức khỏe và giúp họ duy trì lối sống lành mạnh (Kulikovski, Agolli, \& Grougiou, 2011). Hơn nữa, có nhiều nhà nghiên cứu nhận thấy ý thức về sức khỏe là động lực mạnh mẽ để người tiêu dùng mua sản phẩm thực phẩm hữu cơ $(\mathrm{T}$. $\mathrm{B}$. Chen \& Chai, 2010); (Sa'ari \& Koe, 2014); (Huong, 2012). Người tiêu dùng coi một sản phẩm thực phẩm hữu cơ là một yếu tố dinh dưỡng trong việc ngăn chặn con người mắc bất kỳ bệnh nào và đảm bảo bản thân khỏe mạnh (Ahmad \& Juhdi, 2010). Thái độ tích cực đối với thực phẩm hữu cơ đã được (Suh, Eves, \& Lumbers, 2012) thể hiện trong nghiên cứu dựa trên niềm tin của người tiêu dùng rằng các sản phẩm thực phẩm hữu cơ tốt cho sức khỏe con người và họ có thể tự do tiêu thụ nó mà không có bất kỳ nghi ngờ và sợ hãi nào. Do đó, (Wong, Lee, Lin, \& Low, 2012) đã tin tưởng một cách hợp lý rằng người tiêu dùng sẵn sàng hành động lành mạnh là một yếu tố quan trọng quyết định thái độ của người tiêu dùng đối với thực phẩm hữu cơ. Do đó, nghiên cứu sẽ đề xuất:

\section{$H_{2}$ Ý thức về súc khỏe có mối quan hệ đáng kể với ý định mua thực phẩm hũu cơ của người tiêu dùng}

\section{Ý thức về môi trường}

Theo (Ariffin, Yusof, Putit, \& Shah, 2016), mối quan tâm về môi trường có thể được định nghĩa là mức độ e ngại, độ tin cậy và thái độ của một cá nhân đối với môi trường. Theo (Abdul-Muhmin, 2007), có sự chấp nhận rộng rãi giữa các nhà nghiên cứu và các nhà hoạt động môi trường rằng thông qua việc mua các sản phẩm thân thiện với môi trường hoặc các sản phẩm xanh, các sản phẩm có bao bì có thể tái chế hoặc xử lý đúng cách. Người tiêu dùng quan tâm đến môi trường và hạnh phúc của xã hội được thúc đẩy để bảo vệ môi trường bằng cách tìm ra các phương pháp bảo vệ, đổi mới và thực hiện các hành động đúng đắn để bảo vệ môi trường và điều này có thể được thực hiện khi người tiêu dùng trở thành một phần của chiến lược bảo vệ môi trường trong việc lựa chọn môi trường xanh, sản phẩm, sử dụng sản phẩm phân hủy sinh học, tiêu thụ thực phẩm hữu cơ và các sản phẩm khác (Kianpour, Anvari, Jusoh, \& Othman, 2014). Người tiêu dùng có liên quan đến các hoạt động bảo vệ môi trường theo (C. Chen, 2001) đã báo cáo có xu hướng tích cực và chấp nhận tiêu thụ các sản phẩm thực phẩm hữu cơ. Thực phẩm hữu cơ, được sản xuất bằng cách sử dụng các quy trình canh tác tự nhiên, được coi là một chiến lược 
bảo vệ môi trường để giảm ô nhiễm cho môi trường, vì thuốc trừ sâu hóa học và phân bón gây hại cho môi trường, không được sử dụng trong sản xuất (Hassan et al., 2015). Điều này đã buộc người tiêu dùng xanh phải mua sản phẩm thực phẩm hữu cơ vì họ muốn bảo vệ môi trường (Ahmad \& Juhdi, 2010). Do đó nghiên cứu này đã đưa ra giả thuyết:

$H_{3}$ : Ý thức về môi trường có mối quan hệ đáng kể với ý định mua hàng của người tiêu dùng đối với thực phẩm hũu co

\section{Chất lượng}

(Lockie, Lyons, Lawrence, \& Grice, 2004) khuyến nghị rằng các thành phần tự nhiên thường là lý do chính đằng sau việc mua thực phẩm hữu cơ. Thuật ngữ thành phần tự nhiên có liên quan đến thực phẩm chưa qua chế biến, không chứa chất phụ gia hoặc thành phần nhân tạo và không có hóa chất (Lockie et al., 2004). Người tiêu dùng nhận thấy rằng thực phẩm hữu cơ có giá trị và lợi ích và đó là lý do tại sao họ sẵn sàng trả nhiều tiền hơn. (Meier-Ploeger \& Woodward, 1999) tuyên bố rằng 52\% người tiêu dùng Đức trong mẫu của họ sẵn sàng trả nhiều tiền hơn cho rau quả hữu cơ, $34 \%$ cho các sản phẩm động vật hữu cơ và $39 \%$ cho các sản phẩm ngũ cốc hữu cơ. Trong vấn đề nghiên cứu tiêu dùng thực phẩm, nhận thức về chất lượng được coi là vấn đề hàng đầu. Nhận thức về chất lượng thực phẩm an toàn từ người tiêu dùng đóng một vai trò quan trọng trong việc tiêu dùng sản phẩm này (Woodside, Sheth, \& Bennett, 1977). Theo thống kê của siêu thị thực phẩm tự nhiên lớn nhất của Hoa Kỳ - WholeFood vào năm 2014 đã tiến hành một cuộc khảo sát của người tiêu dùng về các lý do khác nhau mua thực phẩm hữu cơ, kết quả thấy rằng $32 \%$ tin thực phẩm hữu cơ có mùi vị tốt hơn thực phẩm thường và $42 \%$ tin rằng chất lượng thực phẩm hữu cơ tốt hơn thực phẩm phi hữu cơ. Cảm nhận về chất lượng đóng một vai trò quan trọng trong việc tiêu thụ thực phẩm hữu cơ tại Thành phố Hồ Chí Minh, tác giả đề xuất giả thuyết:

H4: Cảm nhận về chất lương tác động cùng chiều với ý định mua thực phẩm hưu cơ của người tiêu dùng

\section{Giá}

Nói chung, giá cả và giá trị của một sản phẩm liên quan đến chi phí mua hàng. Theo (Anders \& Moeser, 2008), giá cả và chi tiêu ảnh hưởng đến quyết định lựa chọn thịt hữu cơ. Sự sẵn sàng trả giá cao cho các thực phẩm hữu cơ bao gồm thịt hữu cơ trong số những người tiêu dùng có thu nhập trung bình và cao ở Buenos Aires, Argentina (Lacaze, 2009). (Canavari, Nocella, \& Scarpa, 2003) đã đề cập rằng giá cao được đề xuất cho đào và táo hữu cơ được chấp nhận bởi 65,8\% số người được hỏi trong cuộc khảo sát của họ. Điều đó có nghĩa là giá cả không phải là vấn đề trong việc mua thực phẩm hữu cơ và người tiêu dùng sẽ trả tiền cho thực phẩm nếu họ cho rằng giá cả hợp lý.

\section{$H_{5}$ : Giá cả hợp lý sẽ thúc đẩy ý định mua thực phẩm hũu co của người tiêu dùng}

\section{Phương pháp nghiên cứu}

Vì mục đích chính của nghiên cứu là kiểm tra các yếu tố ảnh hưởng đến ý định mua thực phẩm hữu cơ, một bảng câu hỏi cấu trúc đã được phát triển để thu thập dữ liệu người dân trên 18 tuổi đang sinh sống và làm việc tại Thành phố Hồ Chí Minh thông qua phương pháp phỏng vấn trực tiếp người dân tại các trung tâm mua sắm, siêu thị thông qua phương pháp lấy mẫu thuận tiện. Dũ liệu sau đó được sàng lọc và chỉ 312 phản hồi được coi là đầy đủ và hợp lệ để phân tích dữ liệu. Tác giả thực hiện kiểm định thang đo nghiên cứu bằng hệ số tin cậy Cronbach's Alpha, phân tích nhân tố khám phá EFA, và kiểm định giả thuyết thông qua hồi quy đa biến bằng phần mềm SPSS 20. 


\section{Kết quả nghiên cứu}

\subsection{Mô tả mẫu nghiên cúu}

\section{Bảng 1}

Mô tả mẫu nghiên cứu:

\begin{tabular}{llcc}
\hline & & Tần số & Tần suất $(\boldsymbol{\%})$ \\
Độ Tuổi & Dưới 25 tuổi & 43 & 13,8 \\
& $25-30$ tuổi & 57 & 18,3 \\
& $30-35$ tuổi & 99 & 31,7 \\
& Trên 35 tuổi & 113 & 36,2 \\
Giới tính & Nữ & 172 & 55,1 \\
& Nam & 140 & 44,9 \\
Trình độ học vấn & Dưới THPT & 42 & 13,5 \\
& TN THPT & 81 & 26,0 \\
& TN Cao Đẳng/Đại học & 117 & 37,5 \\
& Sau Đại học & 72 & 23,1 \\
Thu nhập & Dưới 4 triệu đồng & 49 & 15,7 \\
& 4 - 8 triệu đồng & 53 & 17,0 \\
& 8 - 15 triệu đồng & 90 & 28,8 \\
\hline & 15 - 25 triệu đồng & 120 & 38,5 \\
& & $\mathbf{3 1 2}$ & $\mathbf{1 0 0 , 0}$ \\
\hline
\end{tabular}

Nguồn: Kết quả phân tích dữ liệu của nhóm nghiên cứu

Tổng số 312 phiếu khảo sát hợp lệ, trong đó 32,1\% đáp viên ở độ tuổi dưới 30, 31,7\% ở độ tuổi 31 đến 35 tuổi và $36,2 \%$ ở độ tuổi trên 35 . Giới tính đáp viên nữ chiếm $55,1 \%$ và đáp viên nam là 44,9\%. Trình độ học vấn đáp viên chủ yếu là tốt nghiệp Cao đẳng/Đại học (chiếm $37,5 \%)$.

\section{Bảng 2}

Mã hóa thang đo

\begin{tabular}{lll}
\hline Mã hóa & Thang đo & Nguồn tham khảo
\end{tabular}

An toàn thụcc phẩm (AT)

AT1 Tôi quan tâm đến vệ sinh an toàn thực phẩm

(Shaharudin, Pani,

AT2 Tôi luôn quan tâm đến nguồn gốc của sản phẩm

Mansor, \& Elias, 2010)

AT3 Tôi quan tâm tới dây chuyền sản xuất sản phẩm

(Sasaki, Aizaki,

AT4 Tôi nghĩ thực phẩm hữu cơ không chứa hóa chất

Motoyama, Ohmori, \&

Kawashima, 2011) 
Mã hóa

Thang do

Nguồn tham khảo

Ý thức về sức khỏe (SK)

SK1 Tôi quan tâm tới sức khỏe của mình

SK2 Tôi thường nghĩ về các vấn đề liên quan đến sức khỏe

(Shaharudin et al.,

SK3 Tôi nghĩ sức khỏe rất quan trọng trong cuộc sống

SK4 Tôi nghĩ cần phải biết cách ăn uống lành mạnh

SK5 Tôi có thể hi sinh một vài sở thích để bảo vệ sức khỏe của mình

SK6 Tôi quan tâm liệu thực phẩm có tốt cho sức khỏe hay không

\section{Ý thức về môi trường (MT)}

MT1 Tôi thích sử dụng sản phẩm tái chế hoặc có thể dùng lại

MT2 Mọi người khuyên tôi sử dụng thực phẩm hữu cơ để bảo vệ môi trường

MT3 Ô nhiễm môi trường sẽ cải thiện nếu chúng ta cùng hành động

MT4 Tôi đọc mọi thông tin về môi trường

MT5 Công nghệ hiện đại hóa đang hủy hoại môi trường

\section{Chất lượng $(\mathrm{CL})$}

GT1 Tôi nghĩ thực phẩm hữu cơ có chất lượng cao

GT2 Tôi nghĩ thực phẩm hữu cơ có chất lượng cao hơn sản phẩm thực phẩm thông thường

GT3 Thực phẩm hữu cơ tránh rủi ro về sức khỏe

(Shaharudin et al., 2010)

GT4

Tôi nghĩ tôi dùng sản phẩm chất lượng khi tôi sử dụng thực phẩm hữu cơ

\section{Giá cả (GC)}

GC1 Tôi nghĩ giá của thực phẩm hữu cơ cao

GC2 Tôi không ngại chi trả nhiều tiền hơn cho thực phẩm hữu cơ

(Saleki \& Seyedsaleki,

GC3 Một mức giá cả hợp lí rất quan trọng với tôi khi mua thực phẩm 2012) hữu cơ

GC4 Tôi nghĩ thực phẩm an toàn rất mắc

\section{Ý định mua thực phẩm hữu cơ (YD)}

YD1 Tôi có ý định mua thực phẩm hữu cơ

YD2

Tôi sẵn lòng trả nhiều tiền cho thực phẩm hữu cơ để có sức khỏe tốt hơn

YD3 Tôi dự định mua thực phẩm hữu cơ để giảm thiểu những vấn đề xấu về môi trường

YD4 Tôi sẽ chủ động tìm kiếm thực phẩm hữu cơ

YD5 Tôi sẽ mua thực phẩm hữu cơ vì sức khỏe
(Yadav \& Pathak, 2016)

(Sweeney, Soutar, \&

Johnson, 1999)

(Saleki \& Seyedsaleki, 2012) 


\subsection{Kiểm tra độ tin cậy thang đo}

Hệ số Cronbach's alpha là một phép kiểm định thống kê về mức độ chặt chẽ, mức độ hội tụ mà các mục hỏi trong thang đo tương quan với nhau. Kết quả kiểm định thang đo Cronbach's Alpha của các thành phần được trình bày trong Bảng 3 .

\section{Bảng 3}

Kết quả kiểm tra độ tin cậy thang đo của các khái niệm nghiên cứu

\begin{tabular}{|c|c|c|c|c|}
\hline $\begin{array}{c}\text { Biến quan } \\
\text { sát }\end{array}$ & $\begin{array}{l}\text { Trung bình thang } \\
\text { đo nếu loại biến }\end{array}$ & $\begin{array}{c}\text { Phương sai thang đo } \\
\text { nếu loại biến }\end{array}$ & $\begin{array}{c}\text { Tương quan } \\
\text { biến tổng }\end{array}$ & $\begin{array}{l}\text { Cronbach's Alpha } \\
\text { nếu loại biến }\end{array}$ \\
\hline \multicolumn{5}{|c|}{ An toàn thụcc phẩm: Cronbach's alpha $=0,794$} \\
\hline AT1 & 9,39 & 6,28 & 0,54 & 0,77 \\
\hline AT2 & 9,33 & 6,10 & 0,68 & 0,70 \\
\hline AT3 & 9,55 & 6,20 & 0,55 & 0,77 \\
\hline AT4 & 9,41 & 6,10 & 0,65 & 0,72 \\
\hline
\end{tabular}

Ý thức về sức khỏe : Cronbach's alpha $=0,900$

\begin{tabular}{lllll}
\hline SK1 & 15,31 & 18,12 & 0,73 & 0,88 \\
SK2 & 15,31 & 18,08 & 0,70 & 0,89 \\
SK3 & 15,29 & 18,81 & 0,65 & 0,89 \\
SK4 & 15,20 & 17,64 & 0,79 & 0,87 \\
SK5 & 15,30 & 17,38 & 0,76 & 0,88 \\
SK6 & 15,21 & 17,80 & 0,74 & 0,88
\end{tabular}

Ý thức về môi trường: Cronbach's alpha $=0,939$

\begin{tabular}{lllll}
\hline MT1 & 13,51 & 12,05 & 0,89 & 0,91 \\
MT2 & 13,70 & 12,36 & 0,78 & 0,94 \\
MT3 & 13,53 & 12,21 & 0,78 & 0,94 \\
MT4 & 13,53 & 12,03 & 0,87 & 0,92 \\
MT5 & 13,64 & 12,05 & 0,87 & 0,92 \\
\hline
\end{tabular}

Chất lượng: Cronbach's alpha $=\mathbf{0 , 8 6 2}$

$\begin{array}{lllll}\text { CL1 } & 9,68 & 5,86 & 0,69 & 0,83 \\ \text { CL2 } & 9,73 & 5,79 & 0,68 & 0,84 \\ \text { CL3 } & 9,71 & 5,85 & 0,68 & 0,83 \\ \text { CL4 } & 9,71 & 5,41 & 0,79 & 0,79\end{array}$

Giá cả: Cronbach's alpha $=\mathbf{0 , 9 0 0}$

$\begin{array}{lllll}\text { GC1 } & 4,43 & 7,78 & 0,78 & 0,87 \\ \text { GC3 } & 4,20 & 7,48 & 0,80 & 0,86 \\ \text { GC4 } & 4,28 & 6,75 & 0,82 & 0,84\end{array}$




\begin{tabular}{|c|c|c|c|c|}
\hline $\begin{array}{l}\text { Biến quan } \\
\text { sát }\end{array}$ & $\begin{array}{l}\text { Trung bình thang } \\
\text { đo nếu loại biến }\end{array}$ & $\begin{array}{c}\text { Phương sai thang đo } \\
\text { nếu loại biến }\end{array}$ & $\begin{array}{l}\text { Tương quan } \\
\text { biến tổng }\end{array}$ & $\begin{array}{l}\text { Cronbach's Alpha } \\
\text { nếu loại biến }\end{array}$ \\
\hline \multicolumn{5}{|c|}{ Ý định mua thực phẩm hữu co: Cronbach's alpha =0,919 } \\
\hline YĐ1 & 12,46 & 13,79 & 0,78 & 0,90 \\
\hline YĐ2 & 12,50 & 13,33 & 0,84 & 0,89 \\
\hline YĐ3 & 12,60 & 13,66 & 0,74 & 0,91 \\
\hline YĐ4 & 12,50 & 13,89 & 0,78 & 0,90 \\
\hline YĐ5 & 12,47 & 13,39 & 0,83 & 0,89 \\
\hline
\end{tabular}

Nguồn: Kết quả phân tích dữ liệu của nhóm nghiên cứu

Sau khi kiểm định thang đo, tác giả tiến hành phân tích nhân tố khám phá EFA đối với nhóm các biến yếu tố ảnh hưởng đến ý định mua thực phẩm hữu cơ. Hệ số KMO = 0,773> 0,5. Kết quả kiểm định Bartlett's test với mức ý nghĩa < 0,05: Phân tích nhân tố thích hợp với dữ liệu nghiên cứu. Kết quả ma trận xoay nhân tố được trình bày trong Bảng 4.

\section{Bảng 4}

Ma trận nhân tố cho các yếu tố ảnh hưởng đến ý định mua thực phẩm hữu cơ

\begin{tabular}{lccccc}
\hline & \multicolumn{5}{c}{ Nhân tố } \\
\cline { 2 - 6 } & $\mathbf{1}$ & $\mathbf{2}$ & $\mathbf{3}$ & $\mathbf{4}$ & $\mathbf{5}$ \\
\hline SK4 & 0,85 & & & \\
SK5 & 0,84 & & & \\
SK6 & 0,82 & & & \\
SK1 & 0,78 & & & \\
SK2 & 0,77 & & & \\
SK3 & 0,72 & & &
\end{tabular}

MT1

0,91

MT4

0,91

MT5

0,87

MT3

0,83

MT2

0,83

CL4

0,87

CL1

0,80

CL3

0,79

CL2

0,78

GC4 


\begin{tabular}{lccccc}
\hline & \multicolumn{5}{c}{ Nhân tố } \\
\cline { 2 - 6 } & $\mathbf{1}$ & $\mathbf{2}$ & $\mathbf{3}$ & $\mathbf{4}$ & $\mathbf{5}$ \\
\hline AT2 & & & & 0,86 \\
AT4 & & & & 0,85 \\
AT3 & & & $-0,33$ & 0,68 \\
AT1 & 0,33 & & & 0,59 \\
\hline Phần trăm phương sai tích lũy & 30,52 & 44,81 & 56,53 & 65,46 & 73,42 \\
Eigenvalue & 6,71 & 3,15 & 2,58 & 1,97 & 1,75 \\
\hline
\end{tabular}

Nguồn: Kết quả phân tích dữ liệu của nhóm nghiên cứu

Kết quả phân tích EFA cho Ý định mua sản phẩm hữ cơ: Hệ số KMO =0,787 > 0,5; kết quả kiểm định Bartlett's test với mức ý nghĩa $<0,05 \rightarrow$ Phân tích nhân tố thích hợp với dữ liệu nghiên cứu.

\subsection{Phân tích hồi quy đa biến}

Tác giả đã xác định 5 yếu tố ảnh hưởng đến ý định mua thực phẩm hữu cơ, bao gồm: An toàn thực phẩm $(\mathrm{AT})$, Ý thức về sức khỏe $(\mathrm{SK})$, Ý thức về môi trường $(\mathrm{MT})$, Chất lượng $(\mathrm{CL})$, Giá cả $(\mathrm{GC})$ và biến nhân khẩu học giới tính. Tác giả tiến hành phân tích hồi quy đa biến với biến phụ thuộc là Ý định mua thực phẩm hữu cơ (YD), kết quả được trình bày trong Bảng 6 .

\section{Bảng 6}

Kết quả phân tích hồi quy đa biến

\begin{tabular}{lccccc}
\hline \multirow{2}{*}{\multicolumn{1}{c}{ Mô hình }} & \multicolumn{2}{c}{ Hệ số chưa chuẩn hóa } & $\begin{array}{c}\text { Hệ số chuẩn } \\
\text { hóa }\end{array}$ & Giá trị T & Sig. \\
\cline { 2 - 3 } & Bêta & Std. Error & & & \\
\hline Hằng số & $-0,024$ & 0,257 & & $-0,094$ & 0,925 \\
An toàn thực phẩm (AT) & 0,462 & 0,052 & 0,405 & 8,836 & 0,000 \\
Ý thức về sức khỏe (SK) & 0,242 & 0,048 & 0,223 & 5,043 & 0,000 \\
Ý thức về môi trường (MT) & 0,198 & 0,048 & 0,188 & 4,135 & 0,000 \\
Chất lượng (CL) & 0,114 & 0,053 & 0,098 & 2,168 & 0,031 \\
Giá cả (GC) & $-0,073$ & 0,030 & $-0,105$ & $-2,431$ & 0,016 \\
Giới tính [Nũ̃] & 0,136 & 0,075 & 0,074 & 1,818 & 0,070 \\
\hline
\end{tabular}

a. Dependent Variable: Ý định mua thực phẩm hữu cơ

$\mathrm{R}^{2}=0,525 . \mathrm{R}^{2}$ hiệu chỉnh $=0,497$

Nguồn: Kết quả xử lý từ dữ liệu điều tra

Với mức ý nghĩa 5\%, kết quả hồi quy ủng hộ tất cả 5 giả thuyết H1, H2, H3, H4, H5. Kết quả nghiên cứu phù hợp với các nghiên cứu trước, trong đó an toàn thực phẩm là yếu tố quan trọng nhất thúc đẩy ý định mua thực phẩm hữu cơ của người tiêu dùng. Nhiều người tiêu dùng tin rằng thực phẩm được trồng hữu cơ sẽ an toàn hơn và mang lại lợi ích sức khỏe lớn hơn so với 
các lựa chọn thay thế thông thường và có thái độ tích cực đối với các thực phẩm hữu cơ (Beharrell \& MacFie, 1991). Yếu tố quan trọng không kém là sự quan tâm đến sức khỏe, yếu tố này thường được nêu trong việc hình thành thái độ tích cực đối với thực phẩm hữu cơ (Schifferstein \& Ophuis, 1998); (Williams, 2002); (M.-F. Chen, 2007). Thực phẩm hữu cơ có giá trị dinh dưỡng cao hơn vì việc nhân giống và tăng trưởng của thực phẩm hữu cơ đang được thực hiện một cách tự nhiên mà không liên quan đến việc sử dụng hormone và hóa chất. Bên cạnh đó, các sản phẩm thực phẩm hữu cơ được biết đến với chất lượng vượt trội và tươi hơn so với thực phẩm thông thường. Ví dụ, trái cây và rau hữu cơ dường như có độ tươi hơn vì chúng được sản xuất từ các trang trại không có hóa chất và thuốc trừ sâu. Sản phẩm tăng trưởng tự nhiên này cũng có thể cung cấp nhiều chất dinh dưỡng và vitamin tốt cho sức khỏe. Khi những giá trị này được truyền đạt hiệu quả, người tiêu dùng sẽ phát triển ấn tượng tích cực mà cuối cùng có thể biến thành mua hàng, sự hài lòng và lòng trung thành hướng tới sản phẩm. Vì đặc thù canh tác, giá thành của thực phẩm hữu cơ cũng còn khá cao so với cảm nhận của người tiêu dùng, điều này cũng làm cho ý định mua thực phẩm hữu cơ cũng giảm xuống. Cũng có sự khác biệt trong ý định mua thực phẩm hữu cơ giữa nhóm nam và nữ.

\section{Kết luận}

Ý định mua thực phẩm hữu cơ là một vấn đề đáng quan tâm của một quốc gia, nó có thể đảm bảo kinh tế cho một bộ phận không nhỏ người nông dân, nhà sản xuất, thương mại, kích thích tăng trưởng và phát triển kinh tế, đảm bảo sức khỏe cho người dân khi tiêu thụ các thực phẩm sạch. Kết quả nghiên cứu cho thấy người tiêu dùng Thành phố Hồ Chí Minh quan tâm rất nhiều tới vấn đề an toàn thực phẩm, sức khỏe, chất lượng sản phẩm cũng như ảnh hưởng môi trường và giá cả trong ý định mua thực phẩm hữu cơ. Dựa trên kết quả nghiên cứu, thị trường tiêu thụ thực phẩm hữu cơ Thành phố Hồ Chí Minh nên nhắm vào các yếu tố thúc đẩy ý định mua hàng của người tiêu dùng, cung cấp thêm thông tin về lợi ích của thực phẩm hữu cơ đối với an toàn và sức khỏe của cả người tiêu dùng và môi trường. Tiêu thụ thực phẩm phù hợp (thực phẩm hữu cơ) và có chế độ ăn uống phù hợp để mang lại một cuộc sống khỏe mạnh và hạnh phúc là yêu cầu của mỗi người. Thông qua ý thức về sức khỏe, người tiêu dùng sẽ khôn ngoan hơn trong việc lựa chọn thực phẩm hữu cơ phù hợp, có thể mang lại giá trị dinh dưỡng cao hơn so với thực phẩm thông thường trên thị trường. Tát cả điều này có thể xảy ra nếu lợi ích của thực phẩm hữu cơ có thể được truyền đạt tới người tiêu dùng. Càng nhiều thông tin được cung cấp, khách hàng sẽ càng cảm nhận các sản phẩm thực phẩm hữu cơ là thứ có giá trị và đáng mua.

Mặc dù nghiên cứu này đã đóng góp sự hiểu biết tốt hơn về các yếu tố ảnh hưởng đến ý định mua thực phẩm hữu cơ, điều quan trọng là phải thừa nhận những hạn chế đã có trong bài viết này. Có các biến số khác tồn tại và mới có thể ảnh hưởng đến ý định mua thực phẩm hữu cơ đã không được đưa vào mô hình đề xuất. Các nghiên cứu trong tương lai nên kết hợp nhiều biến hơn trong mồ hình để hiểu rõ hơn các yếu tố ảnh hưởng đến ý định mua thực phẩm hữu cơ. Một hạn chế thứ hai là nghiên cứu chỉ thực hiện tại Thành phố Hồ Chí Minh, với cỡ mẫu nhỏ. Trong tương lai, nghiên cứu có thể được nhân rộng cho cỡ mẫu lớn hơn.

\section{Tài liệu tham khảo}

Abdul-Muhmin, A. G. (2007). Explaining consumers' willingness to be environmentally friendly. International Journal of Consumer Studies, 31(3), 237-247.

Ahmad, S. N. B. B., Omar, A. B., \& Rose, R. B. (2015). Influence of personal values on generation Z's purchase intention for natural beauty products. Advances in Global Business Research, 12, 436-445. 
Ahmad, S. N. B., \& Juhdi, N. (2010). Organic food: A study on demographic characteristics and factors influencing purchase intentions among consumers in Klang Valley, Malaysia. International Journal of Business and Management, 5(2), 105-118.

Ajzen, I. (1991). The theory of planned behavior. Organizational Behavior and Human Decision Processes, 50(2), 179-211.

Anders, S., \& Moeser, A. (2008). Assessing the demand for value-based organic meats in Canada: A combined retail and household scanner-data approach. International Journal of Consumer Studies, 32(5), 457-469.

Ariffin, S., Yusof, J. M., Putit, L., \& Shah, M. I. A. (2016). Factors influencing perceived quality and repurchase intention towards green products. Procedia Economics and Finance, 37(16), 391-396.

Beharrell, B., \& MacFie, J. (1991). Consumer attitudes to organic foods. British Food Journal, 93(2), 25-30.

Bernués, A., Olaizola, A., \& Corcoran, K. (2003). Labelling information demanded by European consumers and relationships with purchasing motives, quality and safety of meat. Meat Science, 65(3), 1095-1106.

Canavari, M., Nocella, G., \& Scarpa, R. (2003). Stated willingness to pay for environmentfriendly production of apples and peaches: Web-based versus in-person surveys. Paper presented at the 83rd EAAE Seminar, Chania, Greece.

Cerjak, M., Mesić, Ž., Kopić, M., Kovačić, D., \& Markovina, J. (2010). What motivates consumers to buy organic food: Comparison of Croatia, Bosnia Herzegovina, and Slovenia. Journal of Food Products Marketing, 16(3), 278-292.

Chen, C. (2001). Design for the environment: A quality-based model for green product development. Management Science, 47(2), 250-263.

Chen, M.-F. (2007). Consumer attitudes and purchase intentions in relation to organic foods in Taiwan: Moderating effects of food-related personality traits. Food Quality and Preference, 18(7), 1008-1021.

Chen, T. B., \& Chai, L. T. (2010). Attitude towards the environment and green products: Consumers' perspective. Management Science and Engineering, 4(2), 27-39.

Eagly, A. H., \& Chaiken, S. (1993). The psychology of attitudes. San Diego, CA: Harcourt Brace Jovanovich.

Fishbein, M., Jaccard, J., Davidson, A. R., Ajzen, I., \& Loken, B. (1980). Predicting and understanding family planning behaviors. In Understanding attitudes and predicting social behavior. Upper Saddle River, NJ: Prentice Hall.

Gracia, A., \& de Magistris, T. (2007). Organic food product purchase behaviour: A pilot study for urban consumers in the South of Italy. Spanish Journal of Agricultural Research, 5(4), 439-451.

Harland, P., Staats, H., \& Wilke, H. A. (1999). Explaining proenvironmental intention and behavior by personal norms and the theory of planned behavior. Journal of Applied Social Psychology, 29(12), 2505-2528.

Hassan, S. H., Yee, L. W., \& Ray, K. J. (2015). Purchasing intention towards organic food among generation Y in Malaysia. Journal of Agribusiness Marketing, 7, 16-32. 
Honkanen, P., Verplanken, B., \& Olsen, S. O. (2006). Ethical values and motives driving organic food choice. Journal of Consumer Behaviour: An International Research Review, 5(5), 420-430.

Huong, N. (2012). Key factors affecting consumer purchase intention a study of safe vegetable in Ho Chi Minh City, Vietnam (Master's thesis). International School of Business, HCMC University, Ho Chi Minh, Vietnam.

Kahl, J., Baars, T., Bügel, S., Busscher, N., Huber, M., Kusche, D., . . . Taupier-Letage, B. (2012). Organic food quality: A framework for concept, definition and evaluation from the European perspective. Journal of the Science of Food and Agriculture, 92(14), 2760-2765.

Kianpour, K., Anvari, R., Jusoh, A., \& Othman, M. F. (2014). Important motivators for buying green products. Intangible Capital, 10(5), 873-896.

Kulikovski, V., Agolli, M., \& Grougiou, V. (2011). Drivers of organic food consumption in Greece. Retrieved March 15, 2020, from http://docplayer.net/25279162-Drivers-oforganic-food-consumption-in-greece.html

Lacaze, V. (2009). Sustainable food consumption in Argentine: An estimation of willingness to pay for fresh and processed organic food for consumers in the case Buenos Aires's consumers. Food Science and Technology Abstracts Revista Agroalimentaria, 15(29), 87-100.

Lockie, S., Lyons, K., Lawrence, G., \& Grice, J. (2004). Choosing organics: A path analysis of factors underlying the selection of organic food among Australian consumers. Appetite, 43(2), 135-146.

Meier-Ploeger, A., \& Woodward, L. (1999). Trends between countries. Ecology and Farming, 20(1).

Reid, A. (2000). The face of the web. Retrieved March 15, 2020, from http://www. ipsos-reid. eom/US/SERVICES/pface.cfm

Sa'ari, J. R., \& Koe, W.-L. (2014). The intention to consume organic food among millennial generation. Paper presented at the Proceedings Knowledge Management International Conference, Universiti Teknologi MARA Melaka, Malaysia.

Saleki, Z. S., \& Seyedsaleki, S. M. (2012). The main factors influencing purchase behaviour of organic products in Malaysia. Interdisciplinary Journal of Contemporary Research in Business, 4(1), 98-116.

Sasaki, K., Aizaki, H., Motoyama, M., Ohmori, H., \& Kawashima, T. (2011). Impressions and purchasing intentions of Japanese consumers regarding pork produced by 'Ecofeed,'a trademark of food-waste or food co-product animal feed certified by the Japanese government. Animal Science Journal, 82(1), 175-180.

Scarpa, R., \& Thiene, M. (2011). Organic food choices and protection motivation theory: Addressing the psychological sources of heterogeneity. Food Quality and Preference, 22(6), 532-541.

Schifferstein, H. N., \& Ophuis, P. A. O. (1998). Health-related determinants of organic food consumption in the Netherlands. Food Quality and Preference, 9(3), 119-133.

Shaharudin, M. R., Pani, J. J., Mansor, S. W., \& Elias, S. J. (2010). Factors affecting purchase intention of organic food in Malaysia's Kedah state. Cross-Cultural Communication, 6(2), 105-116. 
Sharma, P. (2011). Country of origin effects in developed and emerging markets: Exploring the contrasting roles of materialism and value consciousness. Journal of International Business Studies, 42(2), 285-306.

Suh, B. W., Eves, A., \& Lumbers, M. (2012). Consumers' attitude and understanding of organic food: The case of South Korea. Journal of Foodservice Business Research, 15(1), 49-63.

Sweeney, J. C., Soutar, G. N., \& Johnson, L. W. (1999). The role of perceived risk in the qualityvalue relationship: A study in a retail environment. Journal of Retailing, 75(1), 77-105.

Verbeke, W., \& Viaene, J. (1999). Consumer attitude to beef quality labeling and associations with beef quality labels. Journal of International Food \& Agribusiness Marketing, 10(3), 45-65.

Voon, J. P., Ngui, K. S., \& Agrawal, A. (2011). Determinants of willingness to purchase organic food: An exploratory study using structural equation modeling. International Food and Agribusiness Management Review, 14(2), 103-120.

Wang, C. L., Li, D., Barnes, B. R., \& Ahn, J. (2012). Country image, product image and consumer purchase intention: Evidence from an emerging economy. International Business Review, 21(6), 1041-1051.

Williams, C. M. (2002). Nutritional quality of organic food: Shades of grey or shades of green? Proceedings of the Nutrition Society, 61(1), 19-24.

Wong, F. V., Lee, M. Y., Lin, X. R., \& Low, S. Y. (2012). A study on the youth attitude toward purchase green products in Malaysia \& Singapore. Retrieved March 17, 2020, from http://eprints.utar.edu.my/716/1/Intention_to_purchase.pdf

Woodside, A. G., Sheth, J. N., \& Bennett, P. D. (1977). Consumer and industrial buying behavior. Amsterdam, Netherland: North-Holland Amsterdam.

Yadav, R., \& Pathak, G. S. (2016). Intention to purchase organic food among young consumers: Evidences from a developing nation. Appetite, 100(96), 122-128.

Yeung, R., \& Yee, W. (2003). Risk reduction: An insight from the UK poultry industry. Nutrition and Food Science, 33(5), 219-229. 